Pedagogía y Saberes n. ${ }^{\circ} 55$ Universidad Pedagógica Nacional

Facultad de Educación. 2021. pp. 41-53

\title{
Lecturas cruzadas entre Freire y Foucault: a propósito de aleturgias escolares y prácticas de libertad*
}

\section{Crossed Readings between Freire and Foucault: On Scholar Alethurgies and Practices of Freedom Leituras cruzadas entre Freire e Foucault: sobre aleturgias escolares e práticas de liberdade}

\section{Para citar este artículo}

Gallo, S. y Espinel, O. (2021). Lecturas cruzadas entre Freire y Foucault: a propósito de aleturgias escolares y prácticas de libertad. Pedagogía y Saberes, (55). https://doi.org/10.17227/pys.num55-13017

* El presente artículo se deriva del Proyecto Interinstitucional Perspectivas metodológicas en el último Foucault. Aportes para una analítica de la educación en derechos humanos y la formación ciudadana C115-030, financiado por la Dirección General de Investigaciones de Uniminuto a través de la $v$ Convocatoria para el desarrollo y fortalecimiento de la investigación en Uniminuto. Proyecto en el que han participado, además de Uniminuto, la Universidad Pedagógica y Tecnológica de Tunja (UPTC) y la Universidad Estadual de Campinas (Unicamp)

** Doctor en educación. Profesor titular de Filosofía de la Educación en la Facultad de Educación de la Universidad de Campinas e investigador becario del Consejo Nacional de Ciencia y Tecnología (CNPq) - Brasil. Correo electrónico gallo@unicamp.br

*** Doctor de la Facultad de Filosofía y Letras de la Universidad de Buenos Aires. Profesor del departamento de Filosofía de la Corporación Universitaria Minuto de Dios - Uniminuto y del Departamento de Posgrados de la Facultad de Educación de la Universidad Pedagógica Nacional (UPN). Miembro del Grupo de Investigación Pensamiento, Filosofía y Sociedad. Correo electrónico: oscar.espinel@yahoo.com 


\section{Resumen}

El artículo de investigación propone continuar la labor de pensar la educación, esta vez, desde el diálogo entre dos autores y dos perspectivas como las expuestas por Michel Foucault y Paulo Freire. Para ello hace uso de las nociones foucaultianas de aleturgia, prácticas de libertad y heterotopía como instrumentos de análisis con el objeto de guiar la discusión, animar las tensiones y pensar posibilidades de fuga para la educación y la escuela. Las diferencias y posturas de cara a conceptos freireanos como prácticas de la libertad y utopía buscan dar forma a nuevas herramientas teórico-metodológicas a fin de problematizar la escuela, la educación y las prácticas hegemónicas desplegadas sobre ellas. En este ejercicio se asume, junto con Freire, la lectura como acto de pensar, de pensarnos e interrogarnos abriendo horizontes de indagación. Una lectura crítica como la reescritura permanente que practicó, vivificó y propició el mismo Freire.

\section{Palabras clave}

aleturgia; educación; heterotopía; prácticas de libertad; utopía

\section{Abstract}

This research article aims to continue the task of thinking education, this time from the dialogue between two thinkers and two different perspectives, such as that presented by Michel Foucault and Paulo Freire. The paper uses Foucault's notions of aleturgy, practices of freedom, and heterotopy as analysis instruments aiming to guide the discussion, animate tensions, and think possibilities of lines of flight for education and school. Differences and positions face to Freire's concepts, such as practices of freedom and utopia seek to produce new theoretical tools and new methodological tools seek to problematize school, education and the hegemonic practices that unfold for them. In this exercise, in conjunction with Freire, reading is assumed as an act of thinking, thinking and interrogating oneself, opening horizons of inquiry. A critical reading such as the rewriting that was made by Freire himself through his life.

\section{Keywords}

aleturgy; education; heterotopy; freedom practices; utopia

\section{Resumo}

Este artigo de pesquisa propõe dar continuidade ao trabalho de pensar a educação, desta vez, a partir do diálogo entre dois autores e duas perspectivas distintas, como aquelas expostas por Michel Foucault e Paulo Freire. Para tanto, faz uso das noções foucaultianas de aleturgia, práticas de liberdade e heterotopia como instrumentos de análise com o objetivo de guiar a discussão, animar as tensões e pensar possibilidades de linhas de fuga para a educação e a escola. As diferenças e posturas face a conceitos freirianos, como prática da liberdade, utopia e liberdade, buscam dar forma a novas ferramentas teórico-metodológicas para problematizar a escola, a educação e as práticas hegemônicas que delas se desdobram. Em tal exercício se assume, conjuntamente com Freire, a leitura como ato de pensar, de nos pensarmos e de nos interrogarmos abrindo horizontes de indagação. Uma leitura crítica como a reescrita permanente que praticou, vivificou e propiciou o próprio Freire.

\section{Palavras-chave}




\section{Introducción}

Este texto tiene por objetivo trazar un diálogo entre dos perspectivas situadas en orillas distintas pero que, por la misma razón, pueden resultar de gran valía en el ejercicio de pensar la educación, la pedagogía y el hacer de maestros y maestras. Las posibilidades del diá-logo se ciernen sobre la diferencia; no se trata de hablar lo mismo para llegar a lo mismo, sino, por el contrario, confrontar-se con lo disímil para hallar nuevas maneras de preguntar y nuevas herramientas para continuar con la investigación. Este, al parecer, es el juego de la crítica y la lectura que el mismo Freire plantea como ejercicio político e inventivo "de ahí que la pedagogía del oprimido, que implica una tarea radical, y cuyas líneas introductorias intentamos presentar en este ensayo implica también que la lectura misma de este libro no pueda ser desarrollada por sectarios" (Freire, 2000, p. 27). En este sentido, la lectura a la que nos estimula el mismo Freire en la invitación a estudiar su libro, es una lectura activa, revolucionaria, pensante, una lectura crítica. En suma, una lectura no para repetir sino para problematizar, interrogar, cuestionar nuestro mundo, lo que somos y, por supuesto, lo que leemos.

Siguiendo esta invitación y asumiendo la lectura como apertura, proponemos un diálogo con esos otros territorios marcados por la extranjeridad en el sentido derridiano - con lo otro y externo a las fronteras propias-, a fin de continuar el compromiso ético-político de pensar la educación. Por esta razón, bajo el propósito de ampliar los horizontes de comprensión y forjar herramientas nuevas de trabajo nos hemos valido, como hilo conductor de esta lectura cruzada, del análisis de la noción foucaultiana de práctica de libertad en conexión con el ámbito de las manifestaciones rituales de la verdad que el filósofo francés denomina "aleturgias". Así pues, la discusión se ubica en el marco de las prácticas educativas haciendo operar las nociones foucaultianas a contramano de las llamadas "pedagogías liberadoras", particularmente, aquella de Paulo Freire.

Para ello, en un primer momento exploraremos un par de tensiones y posibilidades en medio de este diálogo entre los autores. En un segundo momento, ahondaremos en la noción de aleturgia para luego confrontar las nociones de prácticas de libertad y práctica de la libertad. Con estas aproximaciones de base continuaremos con el análisis de la heterotopía como idea de fuga frente a la carga ideológica del concepto de utopía. Finalmente, entendiendo que la educación permanentemente incorpora prácticas de libertad, examinaremos algunas posibilidades de heterotopías a manera de sugerencia y provocación.

\section{Freire y Foucault: tensiones y fugas}

Es necesario, antes de continuar, hacer algunas precisiones en dos escenarios. El primer escenario se nos impone con particular vehemencia: hablar de educación en Latinoamérica y, en especial, en el contexto brasilero, se hace improcedente sin la referencia a un intelectual que, como Paulo Freire, marcó el curso del debate contemporáneo en la región. La Pedagogía del oprimido (1968), escrita en un momento de luchas políticas por la emancipación de una población pobre, oprimida, colonizada y explotada, responde a la perentoria urgencia de encaminar un proceso de superación de una realidad insostenible. Precisamente, uno de los caminos ideados y proyectados con gran esperanza fue la educación del pueblo, la formación de los oprimidos. El título a un texto anterior, publicado en 1965, no deja lugar a dudas respecto a esta concepción política de la educación y las intenciones de la agenda educativa: Educación como práctica de la libertad. Enseñar, señalará Freire, es practicar la libertad; aprender será un acto de libertad; liberarse de las cadenas de la dominación tanto económicas como sociales serán pues el objetivo de la práctica educativa.

Situándose en la tradición de la fenomenología hegeliana pero también influenciado fuertemente tanto por la crítica marxista a la sociedad capitalista como por la teología de la liberación, Freire pensó la educación como un permanente proceso de concientización. Para Freire, la educación es un proceso en el que los sujetos se hacen sujetos de sus propias vidas y de su historia a través de la conciencia de su condición de opresión, negación y deshumanización. El telón de fondo de esta consideración es, desde luego, la conocida metáfora del amo y el esclavo explorada por Hegel en la Fenomenología del Espíritu. ${ }^{1}$ La concientización producida a través de los procesos educativos es el propósito de la crítica junto con la lucha frente a la ideología dominante decidida a mantener la condición de opresión sobre la cual, a su vez, se actualiza y posterga. Es en este sentido que un proceso educativo concientizador y emancipador es, en sí mismo, una práctica de la libertad. Ya tendremos oportunidad de volver sobre ello.

1 Dice Freire haciendo referencia indirecta al célebre pasaje del capítulo IV de la Fenomenología del Espíritu: "No basta saberse en una relación dialéctica con el opresor — su contrario antagónico- descubriendo, por ejemplo, que sin ellos el opresor no existiría (Hegel) para estar de hecho liberados. Es preciso, recalquémoslo, que se entreguen a una praxis liberadora" (Freire, 2000, p. 40). 
Ahora bien, este ejercicio de la crítica, siguiendo las coordenadas freireanas, requiere de la revisión y el estudio riguroso: "solo existe saber en la invención, en la reinvención, en la búsqueda inquieta, impaciente, permanente que los hombres realizan en el mundo, con el mundo y con los otros" (Freire, 2000, p. 73). El estudio y la crítica distan bastante de la entronización de certezas y la reproducción, razón por la cual, más que discipulado o exégesis apostamos por una problematización y actualización de las cuestiones y formas de preguntar de un autor-profesor como Freire. Alejarse de la tendencia a repetir a Freire o dogmatizar (actitud que Freire identificaba con los sectarismos) ${ }^{2}$ significa reconocer también sus límites y procurar ir más allá de él, pensar más allá de él. Pensar con él y no como él. Con este propósito en mente, procuramos transitar estas sendas en compañía de Foucault intentando trazar conversaciones no siempre dirigidas al acuerdo y la armonía. La diferencia, el debate y la confrontación serán entonces el principio orientador de este ejercicio filosófico, pedagógico y crítico.

Un segundo presupuesto del que partimos se aproxima a la analítica foucaultiana para pensar la escuela; esta es una segunda cuestión de fondo alrededor de la cual se teje el presente texto. Aunque la educación no fue un tema central en el trabajo foucaultiano, es un tema con el que se encuentra permanentemente; de hecho, las herramientas que emplea el filósofo han mostrado gran utilidad dentro de los estudios en educación. Así es que acudiremos a Foucault en búsqueda de nociones para el análisis de la escuela y las prácticas de libertad que se derivan de la educación y sus distintas espacialidades.

El filósofo Denis Kambouchner publicó en 2013 el libro L'école: question philosophique en la editorial francesa Fayard, en este trabajo, del que todavía no tenemos traducción ni circulación en español ni portugués, Kambouchner se da a la tarea de rastrear la manera como los distintos filósofos, a lo largo de la historia, abordaron y tematizaron la cuestión de la institución escolar. Justamente, uno de los capítulos se dedica a Foucault y, de manera particular, a Vigilar y Castigar. El estudio de la obra publicada en 1975 por la editorial francesa Gallimard lleva a concluir a Kambouchner que para Foucault la escuela es la

2 Los sectarismos son autoritarios, excluyentes, "negadoras de las dudas, afirmadoras de la verdad poseída por ciertos grupos que se llamaban a sí mismos de revolucionarios". Así lo planteaba el mismo Freire, 25 años después de la publicación de la Pedagogía del oprimido en la relectura que significó la Pedagogía de la esperanza, y continúa en esta misma línea: "reafirmo, como se impone a una Pedagogía de la esperanza, la posición asumida y defendida en la Pedagogía del oprimido contra los sectarismos, siempre castradores, y en defensa del radicalismo crítico" (Freire, 2007, p. 48). máquina disciplinar por excelencia. No obstante, Kambouchner lleva más allá sus análisis y continúa su búsqueda en las labores de Foucault como profesor, esperando encontrar elementos de análisis suficientes para pensar la escuela en el marco de una "era pos-disciplinar". Puesto que Foucault no se dedicó en sus trabajos a pensar, en estricto sentido, una era posdisciplinaria pues sus pesquisas lo llevan hacia la biopolítica y la gubernamentalidad, Kambouchner se dispone a pensar una escuela pos-disciplinar. Es así que, después de comentar algunos puntos que Foucault expone en unas cuantas entrevistas incluidas en los Dits et Écrits, afirma:

\begin{abstract}
Mas también podemos tener en cuenta una particular lección más concerniente a la escuela, con la idea de que donde hay más saber, hay también más libertad y más liberalidad. Si buscamos un funcionamiento escolar que no esté desactualizado, ni que sea autoritario o ciego, lo encontraremos al hacer que el saber que la escuela ofrece a los estudiantes sea más substancial, más dinámico, más general y, por lo tanto, más real. Esto no significa, de ninguna forma, que concibamos la cultura escolar como una totalidad cerrada, sino todo lo contrario. (Kambouchner, 2013, pp. 328-329)
\end{abstract}

El propio Foucault estaba lejos de concebir la escuela como una "cultura cerrada" en sí misma; de hecho, en Vigilar y Castigar evidencia que la maquinaria disciplinar, aunque sea un mecanismo de moldeamiento de los cuerpos, produce efectos positivos como, por ejemplo, hacer posible el aprendizaje, pero "positivos" también en el otro sentido, en el de afirmación, configuración, aparición y positividad. En este sentido, la escuela produce sujetos como el sujeto estudiante, el sujeto docente, el sujeto emprendedor y competitivo, etc. También produce saberes o, al menos, los posibilita, reafirma y pone en circulación: la pedagogía, los saberes disciplinares objetos de enseñanza (biología, matemática, educación física, etc.) y habilita algunos otros como la psicopedagogía, la psicología de la educación, la sociología de la educación, la gestión escolar, etc. Y también da forma y afirma espacios como el aula, el patio de recreo, el laboratorio escolar, la rectoría y coordinación de disciplina, los actos culturales, la emisora estudiantil, el gobierno escolar, entre otros.

Sabemos que, en sus estudios, Foucault no profundiza en la manera como el aparato escolar produce sujetos disciplinados, escolarizados y ciudadanizados pues este no era su objetivo. Sin embargo, no podemos continuar afirmando, tal como tiende a hacerse, que el análisis del filósofo respecto a la institución escolar se limita a resaltar que ella opera como maquinaria de docilización de los cuerpos 
mediante los juegos de poder disciplinar. Es evidente que la escuela opera en esta dirección, pero también es igualmente cierto que estos mismos juegos producen efectos otros que escapan a la docilización y a los mismos diseños del aparato escolar. Ni la vida puede ser atrapada por el aparato institucional, ni los juegos de poder actúan en una sola dirección fija, prescrita o sellada, no existe tal posibilidad. Aceptarlo de esa manera sería caer en cierto determinismo en la historia del que el mismo Foucault rehúye y de la que Freire desdice al destacar el carácter histórico de la acción humana.

Nuestra intención, justamente, se dispone a explorar posibilidades para pensar prácticas de libertad en las instituciones educativas. Afirmar la escuela como escenario de vida, de constitución y reconfiguración en tanto cimentada sobre relaciones humanas, alimentada de infancia y dispuesta al pensamiento crítico-creativo. Espacio de creación y modificación. Para ello, será necesario buscar en otras obras de Foucault herramientas conceptuales distintas que nos permitan pensar de otro modo y preguntar de otro modo. En consecuencia, proponemos pensar y pensarnos desde una noción que no fue tematizada por Foucault y que podríamos nombrar como "aleturgia educativa" o, si se prefiere "aleturgias escolares".

\section{Aleturgias escolares: una noción para pensar la educación}

La noción de aleturgia, como es sabido, fue introducida en el curso de 1980, Del gobierno de los vivos, con el propósito de indicar el tipo de relación intrínseca que existe entre el ejercicio del poder y la manifestación de lo verdadero; una simbiosis entre verdad y poder en el que el uno le sirve de sustento al otro. En sintonía con su hábito de jugar con las palabras, Foucault propone, a partir de un oscuro vocablo del griego antiguo, llamar aleturgia

al conjunto de los procedimientos posible, verbales o no, por los cuales se saca a la luz lo que se postula como verdadero en oposición a lo falso, lo oculto, lo indecible, lo imprevisible, el olvido, [...] no hay ejercicio del poder sin algo parecido a una aleturgia.

(Foucault, 2014, p. 24)

Hay un vínculo entre hegemonía (si por ello entendemos estar en la posición de conducir la conducta de los otros) y aleturgia; en otras palabras, no se puede conducir las conductas de otros sin exhibir una posición de verdad, puesto que ese otro no aceptará ser conducido sino por aquel que está o representa el lugar de la verdad, que expresa lo verdadero en su discurso y se hace, por tanto, emisor de la verdad.
Dos observaciones a este respecto se hacen relevantes. En primer lugar, la existencia de un vínculo que no se reduce en una semejanza lexical entre "aleturgia" y "liturgia". Aleturgia es una especie de liturgia de lo verdadero; una ritualización de la verdad que hace que dicha manifestación se realice en una suerte de teatralización, un performance de la verdad. Foucault inicia su exposición con dos ejemplos usados como antípodas: la sala de justicia del emperador Septimio Severo quien mandó pintar, en el techo de su palacio, el cielo estrellado de su nacimiento como representación del orden astral que dispuso su lugar en el universo, en otras palabras, una estrategia para revelar la verdad de los astros en relación con su destino. El segundo ejemplo empleado por Foucault se refiere a los juegos aletúrgicos envueltos en la tragedia de Edipo Rey, una verdad que configura el destino mismo de Edipo.

En ambos casos, encontramos todo un ritual que se establece en torno a la manifestación de la verdad implicada en los juegos de poder. Otro par de ejemplos en torno a la aleturgia serán expuestos por Foucault en los cursos de El gobierno de sí y de los otros de 1983 (2010a) y El coraje de la verdad de 1984 (2010b), en los cuales se concentra en otra noción metodológica: la parrhesía entendida como decir verdadero. En este caso, la manifestación ritualista de la verdad no es realizada por aquellos que ocupan el lugar privilegiado de poder sino por aquellos que, justamente, desafían dicho poder y su potestad de decir la verdad, aunque ello implique poner en riesgo la propia vida. Dos situaciones sirven de ejemplo en la exposición. El primero de ellos se refiere a aquella escena en la que Platón se enfrenta a Dionisio, el tirano de Siracusa; la segunda escena responde a la imagen de Diógenes, el cínico, quien se enfrenta a la ciudad y sus verdades. Dos formas de aleturgias presentes en ambas situaciones: por un lado, indiscutiblemente, una manifestación de la verdad por parte de aquel que ejerce el poder en contra del filósofo ateniense y, por otro, una manifestación de la verdad a través de la palabra, a través del decir franco que se enfrenta al poderoso. Una aleturgia que desafía las formas dadas.

En segundo lugar, si seguimos la perspectiva adoptada por Foucault, es muy difícil no percibir aleturgias en las más diversas manifestaciones de poder, más allá de las estrictamente políticas en las cuales es sumamente evidente. El filósofo siempre insistió en la centralidad de las relaciones de poder en medio de las acciones humanas. El poder, insiste Foucault, no está solo en la política y las acciones de los políticos; el poder atraviesa, se afirma y actualiza en todos los actos humanos incluso, en sus silencios, resignaciones y 
perplejidades. De modo que, siempre que hay algún tipo de relación entre individuos y grupos, entre lo individual y lo colectivo (materializado de múltiples formas: tradición, principios, ordenamientos, formas culturales, instituciones, creencias, desobediencias, etc.), nos encontramos con relaciones de poder. De suerte que si el poder o mejor, las relaciones de poder, están en todo y en todos lados y, además, si su ejercicio está siempre acompañado de una manifestación de lo verdadero, entonces el fenómeno de las aleturgias es mucho más amplio y abarcador. En otras palabras, la aleturgia no es algo que se limite al ámbito de la política y los "poderosos". Ciertamente, también puede señalarse que lo político es mucho más amplio y abarcador que las acciones de los "políticos". ${ }^{3}$ Si toda acción está signada y atravesada por relaciones de poder podríamos plantear, tal vez de manera apresurada pero no menos cierta, que toda acción también es política y, por tanto, manifestación y actualización de la verdad. Esto llevará a decir a Freire, por ejemplo, que toda educación es política, nunca neutra (Freire, 2007, p. 107).

Hay aleturgias en las manifestaciones religiosas, en el cortejo al amado o a la amada, en los juegos de seducción y, por supuesto, en las relaciones educativas que se establecen entre profesores y estudiantes. Rituales de verdad y manifestación de la y en la verdad. La escuela, la vida escolar, la vida cotidiana, las relaciones interpersonales, las formas de hacer y habitar, todas ellas son modos aletúrgicos en su vínculo multiforme con la verdad y lo verdadero; el sujeto mismo, como lo hemos señalado, es manifestación de la verdad y producción de lo verdadero. Claramente, no se trata de la verdad de los epistemólogos, ni de los científicos o especialistas. Es una verdad del tipo que excede aquellos recortes de lo aceptado como conocimiento (antes bien, sostienen a estos últimos). De hecho, el mismo Foucault aclara que "la manera como esa verdad se manifiesta no es del todo del orden del conocimiento, de un conocimiento formado, acumulado, centralizado, utilizado" (2014, p. 23). Frecuentemente, los límites de lo verdadero

3 Chantal Mouffe logra llamar la atención sobre esta diferencia radical y no siempre tenida en cuenta. Lo propio de lo político es el conflicto, la tensión, el antagonismo. Chantal Mouffe pone en los siguientes términos la distinción entre lo "político" y la "política": "concibo lo 'político' como la dimensión de antagonismos que considero constitutiva de las sociedades humanas, mientras que entiendo a la 'política' como el conjunto de prácticas e instituciones a través de las cuales se crea un determinado orden, organizando la coexistencia humana en el contexto de la conflictividad derivada de lo político" (2011, p. 16). Mientras lo político es lo constituyente, la política es lo constituido. se forjan por efecto de verdades excéntrica, excedentes, espurias, apócrifas. La anécdota de Septimio Severo, por ejemplo, lo ilustra muy bien pues es una "manifestación pura, manifestación fascinante que está destinada, en esencia, no tanto a demostrar o probar algo, a refutar una falsedad, como a mostrar simplemente, a develar una verdad" (Foucault, 2014, p. 23). El campo de la verdad desborda los límites del conocimiento, de lo legítimo y racional para hacerse vigente y actuante en todo el amplio espectro del escenario humano.

Pero volvamos a este tipo de aleturgia que pueblan la cotidianidad escolar y que son las que atraen nuestra atención en este artículo. Deleuze y Guattari (2004, p. 81) señalan en Mil mesetas que la profesora de primaria en-seña (en-signa), es decir, inscribe, induce, introduce, inserta a los estudiantes en un sistema de signos, ${ }^{4}$ la profesora da órdenes, ordena; "la máquina de enseñanza obligatoria no comunica informaciones, sino que impone al niño coordenadas semióticas" (Deleuze y Guattari, 2004, p. 81). Las palabras proferidas por la maestra manifiestan el "verdadero orden" e instalan a los estudiantes en un régimen semiótico específico. Continuando con las pistas dejadas por Foucault, podríamos construir toda una analítica de las aleturgias escolares examinando la arquitectura escolar, la configuración de los espacios, la disposición de los salones de clase, la reglamentación de los tiempos y conductas, la vestimenta de los profesores y estudiantes, las formas de expresión de los profesores y demás actores escolares en consonancia con los espacios y las funciones delegadas dentro de la maquinaria escolar; en fin, toda una serie casi infinita de formas usadas para la manifestación de lo verdadero y la legitimación de las hegemonías que se ejercen alrededor del saber. De este modo, en la manifestación de la verdad, en las prácticas aletúrgicas, la institución escolar se hace efectivamente instituyente.

Es evidente que la escuela es un lugar de manifestación de lo verdadero y un lugar por excelencia de conducción de la conducta. Sin la menor intención de profundizar en ello, por las razones ya expuestas, acudimos a dos ejemplos para mostrar la idea que expresan las nociones de "aleturgias escolares" y

\footnotetext{
4 Unas líneas más adelante dirán los autores franceses "el lenguaje ni siquiera está hecho para que se crea en él, sino para obedecer y hacer que se obedezca" (Deleuze y Guattari, 2004, p. 81).
} 
"aleturgias educativas". ${ }^{5}$ De una parte, pensemos por un momento en la figura del profesor o la profesora. Este personaje encarna en su cuerpo y en su discurso la manifestación de la verdad del saber, tanto del que pretende enseñar como del saber didáctico y pedagógico que orientan su práctica, entre otros. Para que su actuación ${ }^{6}$ sea confiable debe desplegar todo conjunto de ritualidades: un estilo y modos de hablar, una forma de vestir, ciertas posturas corporales y gestualidades, vocabularios y gramáticas especializadas, hábitos y ademanes nodales en su composición aletúrgica. Un profesor necesita inspirar respeto y ese respeto se traduce en términos de saber y poder; justamente, hablamos de autoridad en ese doble juego de poder y saber; una autoridad que deriva en la atención de sus interlocutores, en la confiabilidad y credibilidad. Dicho brevemente, un profesor es respetado porque sabe. Manifestación ritualista de la verdad de un saber que opera como garante de la función desempeñada por el profesor o profesora en un aula de clase.

Otro ejemplo que podemos apuntar a manera de ilustración de las aleturgias escolares puede hallarse en la expansión generalizada y voraz de las evaluaciones masivas y estandarizadas. Evaluaciones masivas que se ponen en sintonía con los criterios y mediciones de ranking y estadísticas locales y globales; razón por la cual se han constituido, en las últimas décadas, en una herramienta biopolítica privilegiada para la gestión de los procesos educativos en escenarios nacionales e internacionales. Este tipo de evaluación, diseñada con el fin de ejercer una regulación estratégica sobre los procesos educativos, controlando lo que los grupos poblacionales deben aprender $(\mathrm{y}$ des-aprender), es la manifestación clara de una verdad global sobre la educación. De modo que quien ya participó de estas estrictas ceremonias de evaluación,

5 Aunque no podremos profundizar en esta distinción, queremos dejar constancia de las diferencias asumidas entre lo educativo y lo escolar o, para ser más precisos, entre educación y escuela. El mismo Freire señala esta diferencia al recordar que la educación excede el aparato escolar mientras que la escuela es una de las formas que asume la educación. De hecho, podríamos continuar señalando el origen moderno (más bien reciente) de los sistemas escolares en occidente que darían lugar a la forma escuela más cercana a como la conocemos en la actualidad. Para ampliar esta idea pueden consultarse los trabajos de Dussel y Caruso (1999) La invención de la escuela, y de Pineau, et ál., M. (2013) La Escuela como máquina para educar. Tres escritos sobre un proyecto de la modernidad.

6 Una actuación que tiene que ver con performance, poner en escena; pero también con hacer actual, con actualizar. En este sentido hablamos de encarnar. Con su voz y su performance, el maestro y la maestra actualizan, afirman y ponen en circulación el saber o conjunto de saberes que incorporan su práctica; algunos más explícitos, intencionados y reflexionados que otros. sea en la función o lugar que sea, fácilmente podrá dar cuenta de toda una serie de ritualidades que, al mismo tiempo que operan en la manifestación de una verdad sobre la educación, ejercen un poder sobre la población involucrada y, a la vez, ejercitan a dicha población en torno a esas relaciones de saber-poder.

\section{¿Prácticas de libertad o prácticas de la libertad?}

Volvamos a la cuestión planteada en el inicio como norte orientador de este ejercicio. ¿Cómo pensar, con Foucault, prácticas de libertad en educación?, ¿qué tipo de vínculos y disonancias se pueden establecer entre estas prácticas de libertad y los planteamientos de un pedagogo como Paulo Freire?, ¿qué diálogos pueden plantearse entre la analítica del poder realizada por Foucault y las pedagogías críticas de carácter emancipador? y ¿qué utillaje metodológico y formas de preguntar resultan de la confluencia de nociones como aleturgia, gobierno por la verdad y prácticas de libertad de cara a las ideas freireanas de práctica de la libertad, crítica y utopía?

Un primer aspecto de la cuestión que debe ser señalado ronda en torno a una diferencia que puede parecer insignificante, sutil e imperceptible pero que resulta de suma importancia para el ejercicio propuesto en la construcción de herramientas de análisis para pensar la educación desde otros ángulos y miradas. Freire pensó la educación como una práctica de la libertad; Foucault, por su parte, pensó en prácticas de libertad. Esto nos lleva, de inmediato, a apreciar tonos distintos tanto en la concepción de libertad como en la de práctica en cada uno de los autores. Dada la amplitud y complejidad en torno a la noción de práctica en Foucault, nos concentraremos aquí, grosso modo, en el uso del término "libertad". Recortes temáticos y conceptuales obligados a fin de poder seguir una ruta expositiva apropiada. ${ }^{7}$

Puede entenderse la formulación de Freire cuando se refiere a práctica de la libertad si se tiene presente la matriz filosófica hegeliano-marxista de la obra del educador brasilero; desde esta perspectiva, la libertad parece ser abordada como una especie de esencia del ser humano, quien además ha de responder a

\footnotetext{
7 Trabajos como los de Roger Chartier (1996), Escribir las prácticas. Foucault, de Certeau, Marin, pueden ayudar en esta línea. Algunas otras discusiones a este respecto han sido recogidas en el libro Educación y pensamiento contemporáneo. Práctica, experiencia y educación compilado por Oscar Espinel (2020) y en el cual participan investigadores de la región latinoamericana de la Red de Investigación en Educación y Pensamiento Contemporáneo (RIEPCO).
} 
una vocación histórica que le es propia. La vocación histórica a ser más (Freire, 2000, p. 32). Es este llamado a su realización lo que le hace ser consciente de su deshumanización por cuenta de las condiciones históricas que le condenan, dominan y anulan como sujeto. La constatación de tales contradicciones y el reconocimiento de sus efectos deshumanizantes, conducen al ser humano a pensar la otra posibilidad, la de la humanización, auténtica vocación histórica. Por esto, afirma Freire

la liberación es un parto. Un parto doloroso. El hombre que nace de él es un hombre nuevo, hombre que solo es viable en y por la superación de la contradicción opresores-oprimidos que, en última instancia, es la liberación de todos. (Freire, 2000, p. 39)

Tal como lo destaca Francisco Weffort en el prefacio a la edición brasilera de la Educación como práctica de la libertad: "la libertad es concebida como el modo de ser o el destino del Hombre, pero por esta misma razón solo puede tener sentido en la historia que los hombres viven" (Weffort en Freire, 1967, p. 6). Aunque el carácter material e histórico de la libertad sea plenamente resaltado, ella es el "modo de ser" de lo humano - del ser humano-, es decir, constituye su esencia. Si la libertad es el ser mismo de lo humano, entonces es preciso realizarla para ser o hacer-se humano; sin libertad no hay humanidad. Es por similares razones que la educación defendida por Freire es una educación para la libertad, una educación liberadora: al realizar la humanidad en el ser humano, se libera de las condiciones políticas e históricas de opresión; así, cuando Freire piensa en la libertad la piensa como sustantivo absoluto.

Bastante diferente es la perspectiva foucaultiana. En una entrevista concedida antes de su prematura muerte en 1984, declara su mesura en torno al tema de la liberación ante la pregunta realizada por Fornet-Betancourt y Gómez-Müller respecto a si las prácticas de sí sobre sí tendrían ese objetivo, la liberación. Foucault, tomando distancia de cualquier postura metafísica y esencialista, critica la idea de una naturaleza o esencia humana que tuviese que ser liberada. Y agrega "a ello obedece el que insista más en las prácticas de libertad que en los procesos de liberación" (Foucault, 1999a, p. 395). Entiende las prácticas de libertad como una forma de regulación de las relaciones de poder, aquello que impide que ellas se tornen absolutas y se transformen en dominación, momento en el cual ni siquiera la resistencia sería posible. De modo que la libertad, lejos de todo universal y entendida como práctica concreta (no como sustancia), es la condición de posibilidad de las relaciones de poder.
En consecuencia, siguiendo la categórica afirmación de Foucault según la cual, "donde hay poder hay resistencia" (2003, p. 116), también podría decirse, dando una vuelta de tuerca adicional, que solo donde hay poder hay libertad. Aún más arriesgado: solo donde hay libertad, por mínima que parezca, hay poder. El siguiente fragmento resulta esclarecedor a este respecto:

Donde determinados factores saturan la totalidad, no hay relaciones de poder; la esclavitud no es una relación de poder cuando el hombre está encadenado (en este caso se trata de una cuestión de relaciones físicas de constricción). Consecuentemente, no hay una confrontación cara a cara entre poder y libertad, que sea mutuamente exclusiva (la libertad desaparece allí donde se ejerce el poder) sino una interrelación mucho más compleja. En este juego, la libertad puede aparecer como la condición para el ejercicio del poder (y al mismo tiempo su precondición, dado que la libertad debe existir para que se ejerza, y también como su soporte permanente, dado que, sin la posibilidad de la resistencia, el poder sería equivalente a la determinación física). (Foucault, 2001, p. 254)

La relación entre poder y libertad, continúa Foucault, no es de antagonismo sino de un "agonismo", de lucha constante, sin que ninguno de los polos pueda vencer de manera definitiva al otro. El poder, como la libertad, son en este sentido, relación y, por tanto, eliminar uno de los puntos en inter-acción significa eliminar, claudicar, finalizar dicha relación, "antes que hablar de una libertad esencial, sería mejor hablar de un 'agonismo', de una relación que es al mismo tiempo recíproca incitación y lucha" (Foucault, 2001, p. 254). Una permanente provocación que alienta la relación mutuamente constituyente.

De este modo, la afirmación de las prácticas de libertad, siempre situadas, circunscritas a unas condiciones históricas y locales es totalmente diferente de una afirmación de la libertad como principio general y global de humanización. No es una libertad esencial (en cuanto sustancia u objeto) a ser recuperada, sino una libertad a ser actualizada, asumida, practicada. Dicho de otra manera, no se trata de un tránsito desde un no ser libre a un estado de libertad efectiva. Por el contrario, se trata de actuar todo el tiempo en la construcción de prácticas de libertad en medio de las situaciones concretas en las que vivimos, produciendo nuevos posibles a partir de las relaciones de poder con otros individuos y otros grupos sociales. El poder se ejerce — afirma Foucault (2001)—, por tanto, podríamos colegir por lo dicho hasta aquí, que la libertad no solo se produce, sino que también se ejerce y se actualiza. Como veremos más adelante, esa 
posición filosófica y política trae consigo consecuencias importantes para pensar las prácticas de libertad en el campo educativo.

Un segundo aspecto de la problemática es que la pedagogía liberadora de Freire, centrada en los procesos de concientización, está orientada por un análisis de carácter ideológico. Como sabemos, Foucault encamina todos sus esfuerzos para desmantelar este tipo de análisis que, según su opinión, reduce las posibilidades de comprensión. El poder no puede ser entendido como un objeto, esencia o bien que se cede o con el cual se realizan transacciones; por tanto, tampoco es "algo" que pueda encontrarse en algún lugar o que sea posesión de alguien (Foucault, 2003, pp. 114-115). El poder es mucho más complejo que la coerción; el poder produce, el poder afirma. Más que un análisis ideológico, el poder convoca un análisis estratégico (Foucault, 2003). En coherencia con este propósito, Foucault despliega un primer desplazamiento hacia los análisis enfocados en las relaciones saber-poder para luego, a partir de los cursos de 1980, dar lugar a un nuevo desplazamiento, esta vez, hacia el gobierno por la verdad (Foucault, 2014).

En la primera clase del curso de 1980, correspondiente al 9 de enero, Foucault presenta tres objeciones generales a la noción de ideología dominante. De manera excesivamente esquemática podrían formularse en los siguientes términos: I) ella está basada en una teoría de la representación (según Foucault, "una teoría mal elaborada"); iI) es una teoría construida a partir de la oposición entre verdadero y falso, entre científico y no científico, realidad e ilusión, por tanto, una teoría que no logra escapar a dicha dualidad; y III) por último, que bajo el término "dominante" la noción de ideología solapa los mecanismos reales de sujeción de los seres humanos y se desentiende de ellos dejando la tarea a otros (Foucault, 2014). Al realizar el desplazamiento hacia la perspectiva del gobierno de los seres humanos por la verdad, Foucault intenta enfatizar la conducción de las conductas de estos seres humanos poniendo en evidencia que son las manifestaciones de lo verdadero las que posibilitan dicha conducción. El ejemplo más ilustrativo que podemos citar a este respecto quizás sea, justamente, aquel que fue analizado en el curso de 1980 y en el ahora conocido cuarto volumen de la Historia de la Sexualidad (Foucault, 2019), a saber: la confesión. Toda una serie de procesos de subjetivación basados en la necesidad de cada uno de manifestar la verdad de sí mismo a través de la confesión; de modo tal que la conducta del devoto cristiano, puesta en manos del director espiritual, resulta guiada por él a través del mecanismo de la verdad y la veridicción.
La perspectiva freireana apunta hacia una dimensión totalizadora y teleológica: la educación como práctica de la libertad significa la superación de una condición de dominación, de opresión en dirección a una condición otra en la cual el sujeto logra emanciparse del juego de dominación dentro del cual se encuentra sometido. La superación de esta situación en la que su existencia es negada significa hacerse sujeto de su propia vida y de su historia. Por esta razón, la práctica educativa que se propone Freire es un proyecto de liberación o, si se nos permite, liberador. Por su parte, tal como hemos visto, en opinión de Foucault no se pueden oponer de manera absoluta y binaria, de un lado el poder y del otro, la libertad; el uno es pre-condición del otro. Tampoco puede darse jerarquía entre poder y libertad; el uno no opera en ausencia del otro. No hay poder absoluto como negación de la libertad, ni libertad absoluta como negación del poder. El poder se hace posible en presencia de libertad y las prácticas de libertad son posibles en medio de relaciones de poder. De hecho, ellas mismas son una forma de relaciones de poder, no solo su resultado. De ahí la precaución al hablar de prácticas de libertad, en plural, siempre múltiples, variables; imprevisibles de manera absoluta, localizadas en el tiempo y en el espacio, singulares; pero, además, siempre provisionales $y$, por tanto, necesariamente actualizadas, renovadas.

\section{Utopía y heterotopías}

Estas precisiones nos llevan a otro lado de la cuestión: a la dimensión utópica de la educación. Un pensador contemporáneo de Foucault, René Schérer, entre otros, puso de relieve el carácter utópico de la educación como parte de un proyecto político. Para tal efecto, se encarga de mostrar que los sistemas educativos son siempre parte integrante de toda propuesta de construcción de una sociedad. ${ }^{8}$ Las utopías, sabemos, abren una perspectiva de futuro, algo a realizar en un momento más allá del presente, las utopías, tal como señaló Ernst Bloch (2007), se sostienen sobre un "principio de esperanza". Dimensión que Freire destacó en la Pedagogía del oprimido en defensa de una educación como práctica de la libertad. Un principio actualizado 25 años después en su libro Pedagogía

\footnotetext{
8 En uno sus ensayos, Schérer comenta:

por lo tanto, la utopía pedagógica es apenas otra cara de la utopía política que, a su vez, no solo está acompañada siempre de pedagogía, sino que ella misma es pedagógica; esto es algo que, por cierto, ya lo sabemos desde Platón. No obstante, esta forma de hablar sería equivocada al sugerir la idea de que la pedagogía pudiese dejar de ser utópica. Ahora se trata, precisamente, de caracterizar la pedagogía, en la generalidad de su propuesta, como utopía. (2009, p. 26)
} 
de la esperanza en el que se propone una relectura y reescritura de aquel libro ideado a finales de los años de 1960. Para el pensador brasilero, la utopía articula denuncia y anuncio, unidas en un compromiso histórico de emancipación y humanización. Afirma en otro de sus libros de mediados de la década de 1970, Acción cultural para la libertad:

la pedagogía que defendemos, concebida en la práctica realizada en un área significativa del Tercer Mundo es, en sí, una pedagogía utópica. Utópica, no porque se nutra de sueños imposible [...] Utópica porque, en contra de la "domesticación" del tiempo, recusa un futuro pre-fabricado que se instalaría automáticamente, independiente de la acción consciente de los seres humanos.

Utópica y esperanzadora porque, pretendiendo estar al servicio de la liberación de las clases oprimidas, se hace y se rehace en la práctica social, en lo concreto, e implica la dialéctica de la denuncia y del anuncio que tiene, a su vez, en la praxis revolucionaria permanente su máximo momento.

Por eso, denuncia y anuncio, en esta pedagogía, no son palabras vacías, sino compromiso histórico. (Freire, 1977, pp. 58-59) ${ }^{9}$

Esa pedagogía utópica implica, por tanto, una apertura permanente hacia un futuro dialéctico o dialectizado. En la medida en que se denuncia una situación de explotación, de manera simultánea, se anuncia la posibilidad de la liberación la cual no será conseguida sin movilización y lucha. "La liberación auténtica, que es la humanización en proceso, no es una cosa que se deposita en los hombres" (Freire, 2000 , p. 84). Sin embargo, la dimensión utópica de la educación implica, necesariamente, un "principio de esperanza" que pone en el futuro la posibilidad de la superación de la condición indeseada del presente.

Foucault es bastante crítico frente a esta idea de utopía debido a la carga ideológica que la nutre. Para Foucault, paradójicamente, las utopías adormecen, acomodan o resignan a los seres humanos en el presente bajo la promesa y la esperanza de un futuro mejor, un futuro por llegar, un estado que aún no está, un proceso en marcha. Este carácter postergador de la utopía conduce a los seres humanos a poner sus esperanzas en la manifestación de una verdad que

9 Preferimos tomar directamente la versión brasilera del texto por cuanto la traducción disponible en español presenta variaciones importantes. se proyecta en el futuro. ${ }^{10}$ Toda perspectiva utópica, en tanto denuncia de una condición y anuncio de otra venidera, no indica un proceso de liberación sino un juego de condiciones a esta nueva situación por venir, una suerte de promesa postergada y siempre esperada. Aunque tuviésemos presente la afirmación de R. Schérer respecto a que la pedagogía es utopía, ¿qué antídoto tendríamos - o sería posible producir- para una utopización de la educación en estos términos?, ¿hasta qué punto, por extraño que parezca, la utopía centra la esperanza en la acción de otro(s)?, ¿hasta qué punto la utopía puede llegar a dilatar la acción y alimentar el conformismo ante una promesa mesiánica por cumplirse?

Para responder a cuestiones como las planteadas, sería necesario acoplar dos conceptos "menores" de Foucault y, por tanto, poco trabajados por el autor. Una vez más, la potencia de la minoridad para hacernos ver, des-ver y apreciar de otras maneras. Un cambio de mirada que implica, a su vez, cierto viraje metodológico. Se trata, como ya se habrá podido intuir, de las nociones de heterotopía y el de prácticas de libertad, ya mencionada. La primera noción, ya presente en el prefacio de Las palabras y las cosas, fue explorada en dos conferencias entre 1966 y 1967. Una de ellas realizada en diciembre de 1966 a través de una entrevista radiofónica (Foucault, 2010) y la segunda, realizada unos meses después en el Círculo de Estudios Arquitectónicos (Foucault, 1999b). Este último, un texto que Foucault no autorizó publicar sino hasta 1984, año de su muerte, luego de ello, Foucault abandonó el concepto y no volvió a referirse a él. La segunda noción, práctica de libertad, ni siquiera llegó a tener los rasgos de una construcción conceptual ya que la noción fue mencionada en una entrevista meses antes de morir. No obstante, la minoridad en el contexto del pensamiento foucaultiano y sus trabajos, parecen sumamente interesantes para, a manera de herramientas conceptuales, inaugurar exploraciones potentes para el campo de la educación y, en particular, para el diálogo aquí propuesto entre dos perspectivas con rumbos propios.

De acuerdo con Foucault, si las utopías consuelan, las heterotopías inquietan y desafían el presente e implican posibilidades concretas de producción, invención e intervención. Así lo afirma explícitamente, unas líneas después de la mención a las utopías en el prefacio a Las palabras y las cosas:

10 En efecto, en el prefacio a Las palabras y las cosas encontramos: "las utopías consuelan: pues si no tienen un lugar real, se desarrollan en un espacio maravilloso y liso; despliegan ciudades de amplias avenidas, jardines bien dispuestos, comarcas fáciles, aun si su acceso es quimérico" (Foucault, 1993, p. 3). 
Las heterotopías inquietan, sin duda porque minan secretamente el lenguaje, porque impiden nombrar esto y aquello, porque rompen los nombres comunes o los enmarañan, porque arruinan de antemano la 'sintaxis' y no sólo la que construye las frases - aquella menos evidente que hace 'mantenerse juntas' (unas al otro lado o frente de otras) a las palabras y a las cosas. (Foucault, 1993, p. 3)

Mientras las utopías se tejen en el espectro cronológico, las heterotopías se ofrecen como noción topológica, mientras las utopías son arrojo al futuro y hacen referencia a un tiempo que aún no es, las heterotopías presentifican, están encarnadas en el aquí y el ahora. Un aquí y un ahora en medio del cual producen diferencias, modificaciones, alteraciones. El aquí y el ahora se hacen tanto condición de posibilidad como objeto y escenario de acción. Por tanto, las heterotopías son rupturas con el tiempo, con la linealidad y causalidad del tiempo cronológico; por ello, las heterotopías también involucran heterocronías en la habilitación de tiempos otros en el seno del tiempo mismo. "Las heterotopías funcionan plenamente cuando los hombres se encuentran en una especie de ruptura absoluta con su tiempo tradicional" (Foucault, 1999b, p. 438). Así pues, la noción de heterotopía arroja una nueva potencia para pensar la educación desde la materialización y presentificación de la acción (educativa) más allá de cualquier registro metafísico. Una educación transgresora del proyecto moderno definido desde la idea de progreso y definido desde una suerte de teleología en la que, muy a pesar de las consignas modernas, el esfuerzo diario haya sentido en la cronología del mundo mejor por venir. Razón por la cual, ideas reguladoras de la acción como progreso, humanización, civilización y prosperidad se amarran a otro concepto, el del por-venir, la misma promesa que alienta la esperanza de una educación utópica y emancipadora.

En pocas palabras, pensar heterotópicamente la educación posibilita la producción de intervenciones concretas en la cotidianidad de la escuela y a partir de ella, es en el presente en donde las prácticas de libertad pueden ser producidas. No implican una superación, un ir más allá o alcanzar una condición que no se tenía antes. No se trata de anuncio, de una propuesta de salvación. No hay "salvación" para el presente; lo que sí podemos constatar es la posibilidad de producir espacios otros en los espacios mismos, en los espacios actuales. La heterotopía nos permite introducir otras claves para pensar la educación, otras rutas para transitarlas y otros espacios para habitarla. Una institución disciplinar, sí; un espacio instituido, sí; pero también un espacio en el que las heterotopías pueden ser producidas en la invención de escuelas otras en la escuela misma, en la escuela vigente.

\section{Algunas provocaciones finales}

En las Primeras palabras de su último libro Pedagogía de la autonomía, publicado en 1996, Freire vuelve a visitar temas ya trabajados e insiste que ello se debe no solo a la relevancia del problema y su inconclusión, sino a la relación de esta con temas que vienen surgiendo en el desarrollo de la reflexión, del pensamiento. Por esta razón, supone que el regreso a los "mismos" problemas no ha de disgustar al lector "sobre todo cuando ese regreso al tema no es pura repetición de lo que ya fue dicho" (Freire, 2008, p. 16). Indudablemente, la lectura y sus rituales son uno -entre muchos otros- de aquellos modos particulares de las aleturgias escolares que aquí nos han convocado y queremos acogernos a ella como ejemplo para respaldar la idea inaugural de este escrito hecho ensayo. Freire nos dará grandes luces sobre ello, aunque aquí solo podamos referir brevemente, a manera de cierre y provocación, uno esos escenarios.

Un primer comentario para continuar en el examen de la lectura como práctica de libertad. El empeñarse en volver sobre aquellos temas ya tratados muestra que no se trata de temas a resolver sino de inquietudes a actualizar. Lo cual deja asomar cierta actitud frente al acto de educar que podríamos catalogar de filosófica. Una relación entre filosofía y pedagogía que no podemos dejar de señalar. Lo más importante no es hallar respuestas a tales preguntas y (pre)fabricar modelos a masificar y universalizar. Quizás lo más importante sea mantener viva la pregunta, mantener vivo el fuego del interés, de la inquietud, frente a lo que hacemos a diario. Juegos aletúrgicos que involucran y afirman el hacer del docente y que, a la vez, se constituyen en escenario de fuga frente a la reproducción mecánica e instrumental que denuncia el mismo Freire.

Y quizás, la imposibilidad de abandonar o ser abandonados por aquellas preguntas, también se deba a que en educación no hay modelos, ni situaciones, ni escenarios, ni problemas idénticos. Todo es siempre nuevo. La educación está llena de diferencia, novedad y, por tanto, de incertidumbre, incerteza. La educación, podríamos decir con Walter Kohan (2016), es un viaje que nos exige explorar y aprender permanentemente. De tal suerte que no solo el pensar y re-crear el hacer docente se configuran como prácticas de libertad, sino que el aprender mismo, el retomar lo visitado para darle nuevas formas, el acto de leer para preguntar de otros modos, toman cuerpo como puntuales y singulares escenarios de libertad, de multiformes e íntimas prácticas de libertad.

Ello también se relaciona con la lectura crítica de la que ya nos hablaban las palabras introductorias de la Pedagogía del oprimido. Una lectura para seguir 
pensando, que no busca certezas sino formas de preguntar, formas de actualizar la pregunta. Y es la lectura, justamente, uno de esos espacios en el que estudiantes y docentes se encuentran. "No hay docencia sin discencia", es el título que Freire da al primer capítulo de la Pedagogía de la autonomía. Podríamos alternar el neologismo freireano discencia por estudio, como aquello propio del que aprende. Y es interesante esta aclaración que nos hace Freire, porque en medio de la turbulencia de los días, el estudio va siendo desplazado. Es algo que se abandona o se deja para "después". Un "después" que tarda en llegar y que, generalmente, nunca llega. A tal punto que el estudio tiende a hacerse excepción, algo que siempre se aplaza para poder atender lo más urgente.

Es curioso que el profesor que invita a la lectura, al estudio, abandone, de vez en cuando, el estudio y la lectura, siempre atareado y cargado de urgencias olvida aquello que lo hace docente: el estudio. Pero esto mismo tiende a sucederle a los estudiantes, siendo estudiantes, abandonan el estudio. Lo dejan para después porque primero está responder a las tareas, consultas, escritos y cuadros de las clases, la lectura se desdibuja y se hace una cuestión burocrática, formal, protocolaria, superficial. Algo que se hace (si es que se hace) por cumplir, por responder a una exigencia externa, disciplinaria, punitiva. Y eso, lo sabemos, no es leer; como tampoco es escribir el diligenciar formularios o inundar páginas de reportes e imposturas. Quizás se asemeja más a una revisión de textos e información para entregar informes. ¡Qué paradójico! En la escuela todo el tiempo nos relacionamos con textos, con escritos y parece ser que lo que menos hacemos es leer, somos estudiantes y lo que menos hacemos es estudiar. ¿por qué no pensar una escuela en la que la lectura y el estudio sean posibles como experiencia y no como trámite burocrático para conseguir otros fines o responder a mediciones ajenas?, ¿por qué no cimentar, desde el aquí y el ahora del aula de clase, heterotopías en las que la lectura, el estudio y el aprender se hagan por sí mismos y no en función de algo por-venir?, ¿por qué no hacer de la escuela esa heterotopía que recuerda del término griego scholé como tiempo del ocio, tiempo de la atención, tiempos de la suspensión, tiempo del estudio?

Finalmente, podríamos cerrar mencionando con Freire el carácter ético de la práctica educativa en cuanto práctica específicamente humana. Advierte Freire:

al sujeto ético no le es posible vivir sin estar siempre expuesto a la transgresión de la ética. Por eso mismo, una de nuestras peleas en la Historia es exactamente esta: hacer todo lo que podamos a favor de la eticidad, sin caer en el moralismo hipócrita, de reconocido sabor farisaico. (Freire, 2008, p. 19) [Énfasis agregado]

Muchos elementos y cuestiones por comentar en este fragmento; sin embargo, solo nos quedaremos con aquella idea de "transgresión de la". Una invitación a transgredir esos sistemas aparentemente terminados, definidos, cerrados denotados con la expresión "de la”. Esos sistemas de reglas, nos dirá Freire, en tanto producto humano, son históricamente construidos y, por tanto, provisionales. Pero, además de ello, nos invita a una transgresión que resulta en una actitud, la eticidad. Una actitud transgresiva que se asemeja a aquello que Foucault entiende como ética en tanto modificación, práctica de sí y cuidado de sí. Una permanente atención resumida en el principio griego de epimeleia heatou traducida como inquietud de sí. Una inquietud permanente y actualizada en la que se juegan las prácticas de libertad y que propicia nuevos y permanentes escenarios de aleturgias escolares y educativas. Prácticas que lejos de ser individualistas, insulares o solipsistas, requieren, se acompañan y producen en relación con otros y con lo otro. Todo ello nos hace pensar, una vez más, en la educación como un escenario poblado de polifónicas prácticas de libertad. Pues bien, ¿qué prácticas de libertad se asocian a la práctica educativa?, ¿qué tipo de práctica es la práctica educativa?, ¿es posible pensar e idear heterotopías desde nuestras aulas? Y ¿qué tiempos de posibilidad nutren nuestra historia, nuestra escuela, nuestro hacer?

\section{Referencias}

Bloch, E. (2005). El principio esperanza. Editorial Trotta

Chartier, R. (1996). Escribir las prácticas. Foucault, de Certeaur, Marin. Ediciones Manantial.

Deleuze, G. y Guattari, F. (2004). Mil Mesetas. Capitalismo y esquizofrenia. Pretextos.

Dussel, I. y Caruso, M. (1999). La invención del aula. Una genealogía de las formas de enseñar. Ediciones Santillana.

Espinel, 0. (Comp.). (2020). Educación y pensamiento contemporáneo. Práctica, experiencia y educación. Uniminuto.

Foucault, M. (1993). Las palabras y las cosas. Siglo XXI.

Foucault, M. (1999a). La ética del cuidado de sí. En Obras esenciales. Volumen 3. Estética, ética y hermenéutica (pp. 393-416). Paidós.

Foucault, M. (1999b). Espacios diferentes. En Obras esenciales. Volumen 3. Estética, ética y hermenéutica (pp. 431442). Paidós. 
Foucault, M. (2001). El sujeto y el poder. En H. Dreyfus y P. Rabinow (Eds.), Michel Foucault. Más allá del estructuralismo y la hermenéutica (pp. 241-259). Nueva Visión.

Foucault, M. (2003). Historia de la Sexualidad 1. La voluntad de saber. Siglo XXI.

Foucault, M. (2010). El cuerpo utópico. Las heterotopías. Nueva Visión.

Foucault, M. (2014). El gobierno de los vivos. Fondo de Cultura Económica.

Foucault, M. (2019). Historia de la Sexualidad 4. Las confesiones de la carne. Siglo XXI.

Freire, P. (1967). Educação como prática da liberdade. Paz e Terra.

Freire, P. (1977). Ação cultural para a liberdade - e outros escritos. $2^{\mathrm{a}}$ ed. Paz e Terra.

Freire, P. (2000). Pedagogía del oprimido. Editorial Siglo XXI.
Freire, P. (2007). Pedagogía de la esperanza. Editorial Siglo XXI.

Freire, P. (2008). Pedagogía de la autonomía. Editorial Siglo XXI

Kambouchner, D. (2013). L'École: question philosophique. Fayard.

Kohan, W. (2016). El maestro inventor. Simón Rodríguez. Ediciones del Solar.

Hegel, G. (1966). Fenomenología del Espíritu. Fondo de Cultura Económica.

Mouffe, Ch. (2011). En torno a lo politico. Fondo de Cultura Económica.

Pineau, P., Dussel, I. y Caruso, M. (2013). La Escuela como máquina para educar. Tres escritos sobre un proyecto de la modernidad. Editorial Paidós.

Schérer, R. (2009). Infantis. Charles Fourier e a infância para além das crianças. Autêntica. 